\title{
Hybrid Fireworks Algorithm for the Conversion of Planar Curve to B-Spline Curve
}

\author{
K. Sreenivasa Reddy, D. Sai, B. Vijay Sai, E. Kamal Raju, D. Praveen Kumar
}

\begin{abstract}
Point sampling is essential for the conversion of planar curves to B-spline curves in geometric modelling applications. Conversion of parametric curve to B-Spline curve is often required as the latter provides the flexibility sought by the designer. Sampling methods generally ignores the feature points, which indicates the curve profile intuitively and they require user intervention. There is a need for generalized point sampling algorithm to capture the original shape of the planar curves. Auxiliary points are also needed which helps to define the curve and gives the better conversion into B-Spline curve. In this work, we developed a generalized point sampling algorithm based on fireworks algorithm for the conversion of parametric curve to $B$-spline curves. It is used curvature-based information to identify the feature points, while Fireworks algorithm is used for the identification of the auxiliary points. Developed algorithm was tested against curves with irregular shapes and cusps with no need of user intervention to tune the algorithm for conversion.
\end{abstract}

Keywords: Curve conversion, B-Spline, fireworks algorithm, parametric curves.

\section{INTRODUCTION}

Computer aided design and geometric modelling applications requires the polynomial representation of curves. Usage [1]. Conversion of parametric curves to B-Spline curves is often required as the latter provides the flexibility sought by the designer. Most of the times implicit curves and parametric curves cannot be converted into B-Spline curves with desired accuracy. The conversion of one form of curve to B-Spline curve is an approximation of a given curve to $\mathrm{B}$-Spline curve [2]. Conversion of planar curves to B-Spline curve need a generalized frame work. Many algorithms were developed and implemented by researchers for point sampling to aid in curve conversion.

B-Spline curves are widely used in free form curve and surface design as they provide local and global control over the curve shape. This paper focuses on point sampling to

Revised Manuscript Received on April 04, 2020.

* Correspondence Author

K Sreenivasa Reddy*, Department of mechanical, Godavari Institute of Engineering and Technology, Rajahmundry 533296, India. Email: deptworks.giet@gmail.com

D Sai, Department of Mechanical, Godavari Institute of Engineering and Technology, Rajahmundry 533296, India. Email: saidarru@gmail.com

B Vijay Sai, Department of Mechanical, Godavari Institute of Engineering and Technology, Rajahmundry 533296, India. Email: saivijay1779@gmail.com

E Kamal Raju, Department of Mechanical, Godavari Institute of Engineering and Technology, Rajahmundry 533296, India.

D Praveen Kumar, Department of Mechanical, Godavari Institute of Engineering and Technology, Rajahmundry 533296, India.

(C) The Authors. Published by Blue Eyes Intelligence Engineering and Sciences Publication (BEIESP). This is an open access article under the CC BY-NC-ND license (http://creativecommons.org/licenses/by-nc-nd/4.0/) convert planar parametric curve with irregularities such as cusps into B-Spline curves. Point sampling involving identification of feature points, which indicate the curve shape intuitively is proved to be effective in earlier works. Feature points used are inflection points and extreme

curvature points. They are identified based on curvature function values. In addition to the feature points, other points, called conventionally as auxiliary points are also required for accurate conversion.

Proposed algorithms in literature used weighted characteristic functions involving curvature and arc length functions. This approach requires the tuning of weight value for accurate conversion. So, user expertise is required for the conversion of curves with irregularities such as cusps, making the algorithm less general.

Swarm intelligence algorithms proven valuable for the problems of optimization and many are applied to curve fitting problems also. Fireworks algorithm is getting more prominence in recent years for the optimization problems [3]. The proposed method uses a hybrid algorithm, which identifies both inflection, extreme curvature points based on the curvature values and auxiliary points through fireworks algorithms.

\section{LITERATURE REVIEW}

Park [4] proposed an error bound approximate method for the conversion of planar curve to B-Spline curve. In that polygonal approximation is employed to get accurate conversion. An equal weight characteristic function involving arc length and curvature function is used to determine the sampling points for the conversion of parametric curves and surfaces to NURBS curves and surfaces by Pagani et al.[5]. An approximate B-Spline curve for set of data points is fitted by using discrete curvature values at the data points by $\mathrm{Li}$ et al.[6]. They used the curvature information to adjust the knot placement to get the better results. $\mathrm{L} \mathrm{Lu} \mathrm{[7]} \mathrm{proposed} \mathrm{weighted} \mathrm{progressive}$ iteration approximation method for the data points and compared the results with progressive iteration approximation. Weighted progressive iteration approximations scheme is used by $\mathrm{L} \mathrm{Lu}$ [8] to gradually adjust the control points for approximation of given planar curves with rational Bezier curves. D.J Walton et al. [9] numerically obtained the curvature extreme values for polynomial cubic curves having cusps, self-intersection. Square distance method integrated with fast marching method is employed for control point adjustment to get approximating B-Spline curve for data points by $\mathrm{H}$ Yang et al. [10]. 
A Razdan [11] proposed algorithm to determine the number of points required for the conversion and used arc length and curvature functions to obtain the interpolation points. Deng et al. [12] implemented least squares progressive iteration algorithm for approximating given curves with B-Spline curve and presented method to compute the weight values.

Laube et al. [13] employed support vector machines for taking sequential number of points that are located on the curve and find the feature points where that point gives better approximation error. Kosters [14] employed uniformly subdivided parametric space along with angle parameterizations for curve approximations with B-Spline curves. Park et al.[15] proposed a method for approximate NURBS curve compatibility based on multiple curve refitting.

From the literature review, methods proposed for the curve conversion employs weighted functions and hence requires user intervention. There is a need for development of algorithm to mimic the user intervention in curve conversion problems. This paper presents hybrid fireworks algorithm applicable to planar parametric curves with irregularities like cusps etc.

\section{HYBRID METHOD FOR CURVE CONVERSION}

Curve conversion represents an optimization problem in which the sampling points are selected to minimize the error associated with conversion of given parametric curve to B-Spline curve. The conversion error used in the present work is root mean square error (RMSE) and is given as

$$
E=\sqrt{\frac{\sum_{i=1}^{n}\left(P_{i}-\sum_{j=1}^{n p t s} N_{j, k}\left(u_{i}\right) \times B_{j}\right)}{n}}
$$

Where

$$
\begin{gathered}
n=\text { Number of sampled points } \\
\text { npts }=\text { Number of control points } \\
k=\text { order of the } B-\text { Spline curve } \\
u=\text { parameter value } \\
N_{j, k}=B-\text { Spline basis functions }
\end{gathered}
$$

\section{A. Feature Points}

Feature points are considered for sampling as they intuitively represent shape of the curve. Basically, the feature points are of two types: Inflection points and Extreme curvature points. Proposed method uses Algorithm 1 to identify the inflection and extreme curvature points.

\section{Algorithm 1}

Determine curvature function $(\rho)$ value at uniformly initiated parameter values $u_{i}$.

for each $u_{i} \varepsilon$ \{uniformly spaced parameter values\}

$$
\text { if } \rho(i)=0 \text { and } \rho(i-1) \times \rho(i+1)<0
$$

select point as inflection point.

else if $\rho(i) \neq 0$ and $\rho(i) \times \rho(i+1)<0$

fit parabola $\rho(u)$ by interpolation through the points

$\left(u_{i}, \rho_{i}\right),\left(u_{i+0.5}, \rho_{i+0.5}\right)$ and $\left(u_{i+1}, \rho_{i+1}\right)$

Inflection points is the root of equation $\rho(u)$.

$$
\text { end if }
$$

end for

for each $u_{i} \varepsilon$ \{uniformly spaced parameter values\}

if $\rho(i)</>\rho(i+1) \& \rho(i)</>\rho(i-1)$

fit parabola $\rho(u)$ by interpolating through the points

$\left(u_{i-1}, \rho_{i-1}\right),\left(u_{i}, \rho_{i}\right)$ and $\left(u_{i+1}, \rho_{i+1}\right)$

$$
\begin{aligned}
& \text { extreme curvature point is the root of equation } \rho^{\prime}(u)=0 \\
& \text { end if } \\
& \text { end for }
\end{aligned}
$$

\section{B. Auxiliary Points}

Auxiliary points improve the conversion accuracy and are conventionally selected by using characteristic functions. These characteristic functions involve arc length and curvature functions in weighted form. Those functions work well with regular shaped planar parametric curves. But requires user intervention in case of irregular shaped curves. Algorithm proposed in this paper selects auxiliary points through fireworks algorithm. Fig. 1 shows the flow chart of the hybrid fireworks algorithm for the point sampling.

Each firework in the algorithm is a vector of size equal to number of sampling points required. Except parameter values corresponding to the feature points, remaining are initialized randomly. This makes the algorithm more general for any shape of the curve. The optimization problem (2) involves adjusting the randomly initialized parameters, so that the error of conversion given by (1) is minimized. For each firework, number of sparks are generated according to (3), which ensures a greater number of sparks for better firework. Equation (4) gives the amplitude of the explosion, so that sparks are generated closely for the better firework.

$$
\text { Minimize } E(u) \in R, \quad u_{\min } \leq u \leq u_{\max }
$$

The number of sparks general for a firework $s_{i}$, is expressed as

$$
S_{i}=S \frac{E_{\max }-E_{i}+\varepsilon}{\sum_{i=1}^{n}\left(E_{\max }-E_{i}\right)+\varepsilon}
$$

Where $\mathrm{S}$ is the parameter controlling the total number of sparks generated for that explosion generation. $E_{\text {max }}$ is the maximum value of the objective function which is the objective function value of the bad firework.

$$
A_{i}=A \frac{E_{i}-E_{\min }+\varepsilon}{\sum_{i=1}^{n}\left(E_{i}-E_{\min }\right)+\varepsilon}
$$

Where

$$
\begin{aligned}
& A=\text { Maximum amplitude of the explosion } \\
& E_{\text {min }}=\text { Minimum value of error }
\end{aligned}
$$




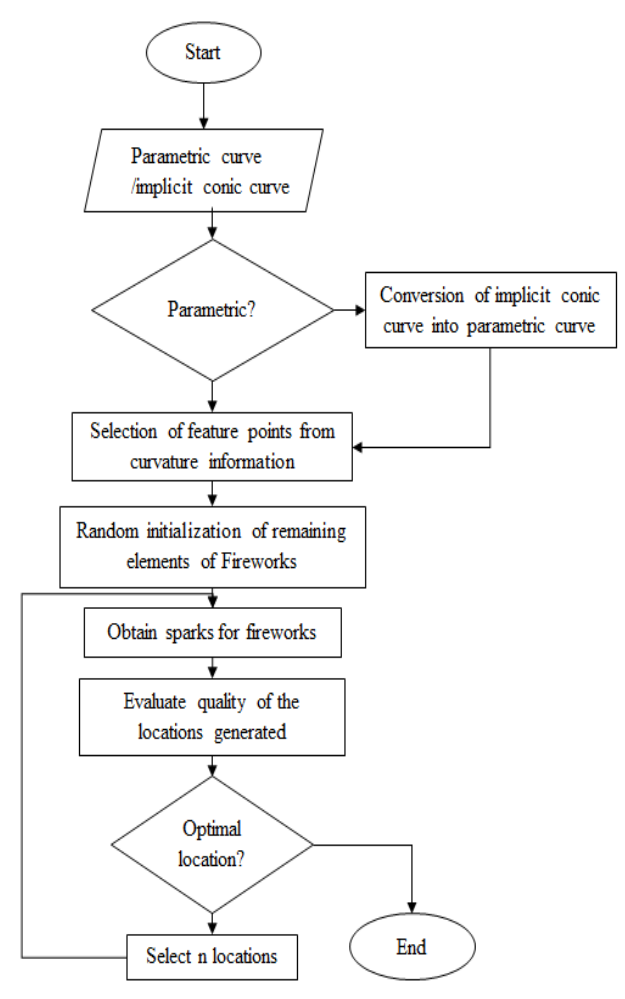

Fig. 1.Hybrid Fireworks algorithm for point sampling

Two parameter sets must be decided before searching for the solution. Number of control points and order of curve are the parameters belongs to the approximating B-Spline curve and are depending on the complexity of the curve to be converted. Like other naturally inspired algorithms, values for the control points for Firework algorithm influence its performance. In this work, control parameters are set based on results of both theoretical and test cases from literature. Large number of sampling points improves the conversion accuracy but increases the CPU time. Values in the range 20-40 found satisfactory from the test cases. Number of sparks and number of fireworks are the parameters which control the number of iterations required and hence the CPU time. Through test cases, range for those parameters are 3-5 and 2-10 respectively. Amplitude of vibration is selected in the range 0.01 to 0.03 , in order to maintain diversity and to avoid ill conditioning of the algorithm.

\section{RESULTS AND DISCUSSIONS}

Proposed algorithm is tested against several examples. Chosen examples demonstrates the irregularities of the curve and as shown in Table 1, can be converted accurately by the method presented. All the parametric curves are converted into a cubic B-Spline curves though curves of higher order can also be obtained. All computations have been performed on Intel ${ }^{\circledR}$ Core ${ }^{\mathrm{TM}}$ i7 processor with 8GB of RAM. The source code has been implemented on MATLAB ${ }^{\circledR}$.

Table- I: Curve conversion results for examples

\begin{tabular}{|c|c|c|c|c|}
\hline Example & $\begin{array}{c}\text { No. of } \\
\text { Sampled } \\
\text { points }\end{array}$ & RMSE & $\begin{array}{c}\text { Control } \\
\text { polygon } \\
\text { Vertices }\end{array}$ & Order \\
\hline 1 & 20 & 0.007 & 6 & 4 \\
\hline 2 & 20 & 0.0172 & 6 & 4 \\
\hline
\end{tabular}

Retrieval Number: D7632049420/2020@BEIESP

DOI: 10.35940/ijeat.D7632.049420

Journal Website: www.ijeat.org

\begin{tabular}{|l|l|l|l|l|}
3 & 20 & $2.80 \mathrm{E}-16$ & 5 & 4 \\
\hline 4 & 20 & $6.56 \mathrm{E}-16$ & 5 & 4 \\
\hline 5 & 20 & $3.17 \mathrm{E}-15$ & 5 & 4 \\
\hline
\end{tabular}

Sampling points obtained by the proposed algorithm for example parametric curve (5) are shown in fig. 2 as circles on blue line. This example shows the applicability of the algorithm for the curves having self-intersection. The approximating B-Spline curve (red line) along with sampling points is shown in fig. 3. RMSE value reported in table shows the capability of the algorithm, and is in agreement with the visual result.

\section{A. Example-1}

The parametric curve equation

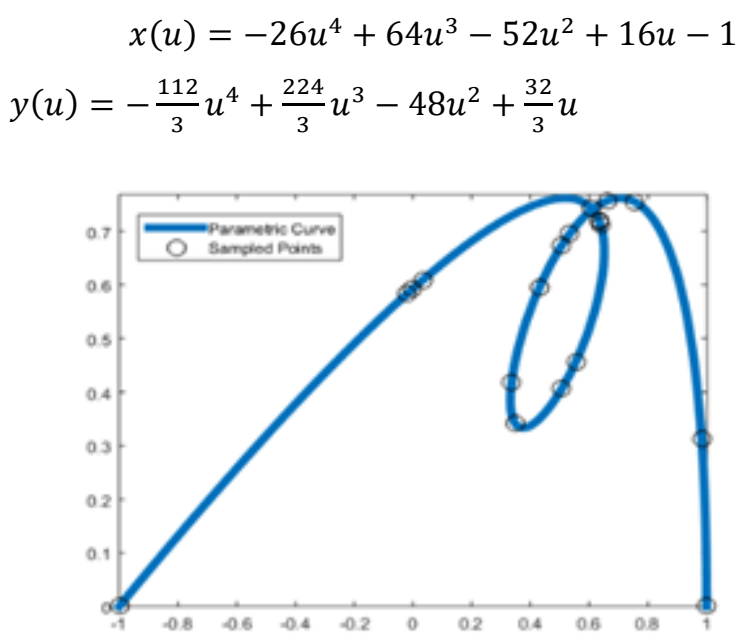

Fig. 2.Parametric curve for Example-1

Example 2 uses a parametric curve demonstrating all the feature points i.e. extreme curvature and inflection points. Fig. 4 shows the curve along with sampling points. The approximating B-Spline curve fig. 5 does not pass through the sampling points and the best results obtained are reported in Table I. With increased number of iterations, the conversion accuracy can be improved.

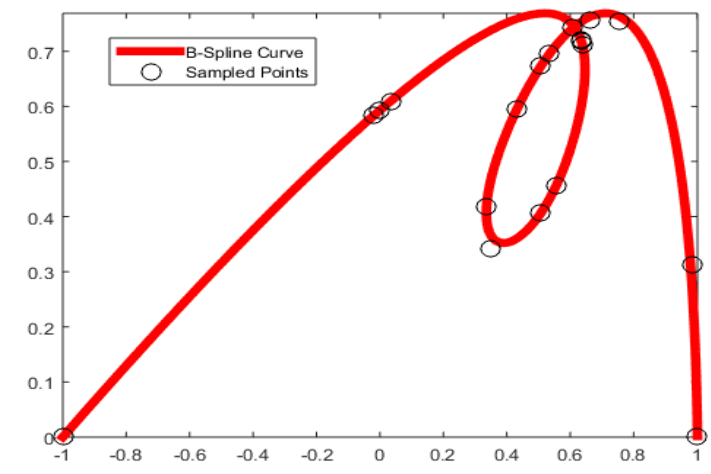

Fig. 3.B-Spline curve for Example-1

B. Example-2

The parametric curve equation

$$
x(t)=u
$$


$y(t)=u-0.5 u^{2}+0.2 \sin (12 u)$

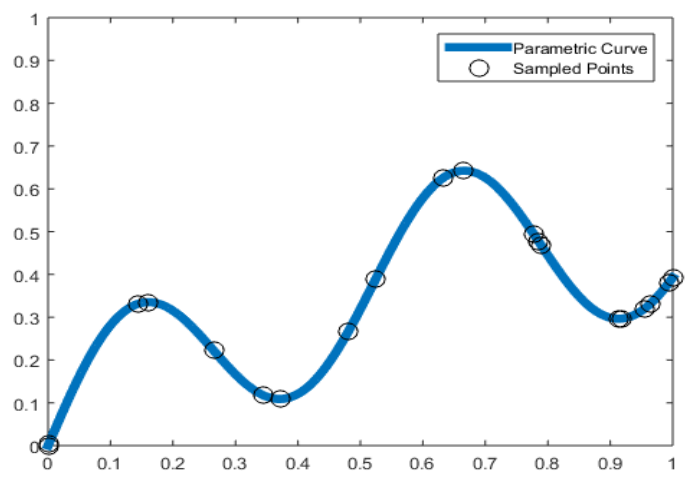

Fig. 4. Parametric curve for Example-2

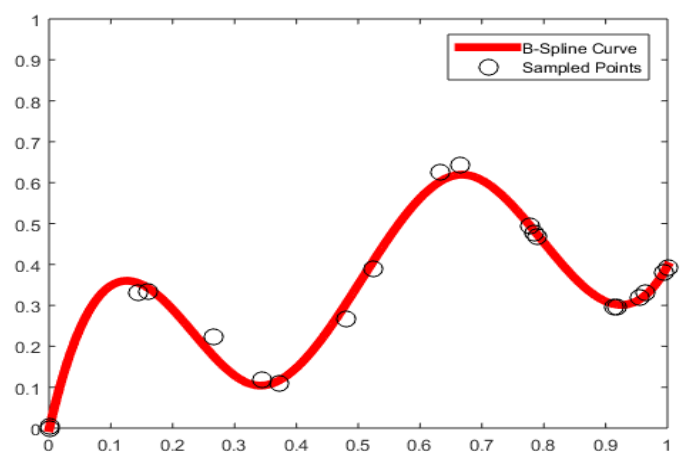

Fig. 5. B-Spline curve for Example-2

\section{Example-3}

The parametric curve equation

$$
y(t)=u^{3} \quad x(t)=u^{2}
$$

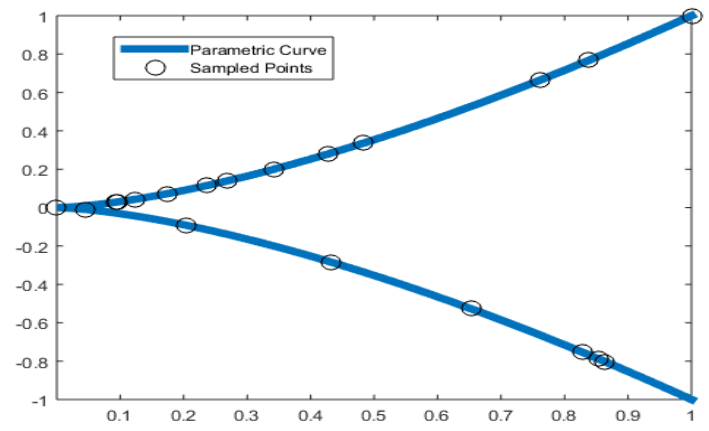

Fig. 6. Parametric curve for Example-3

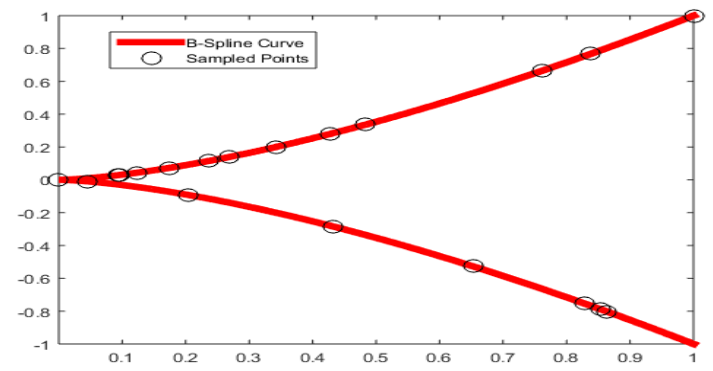

Fig. 7. B-Spline curve for Example-3

D. Example-4

The Parametric curve equation $x(t)=u^{2}-1$

$y(t)=u^{3}-u$

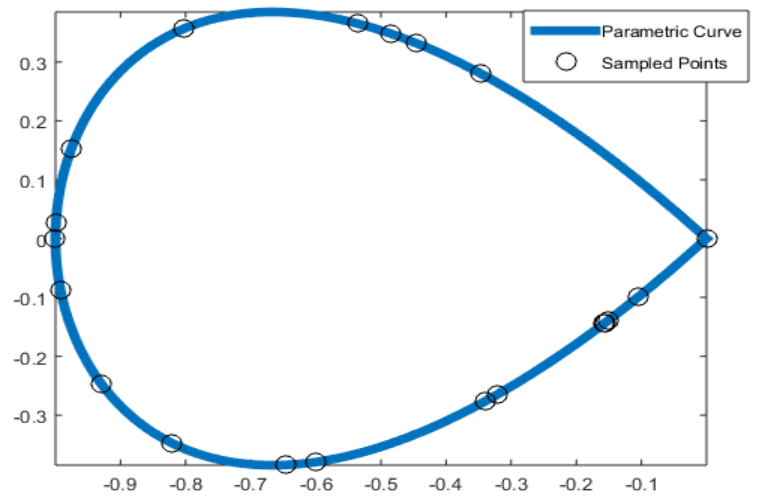

Fig. 8. Parametric curve for Example-4

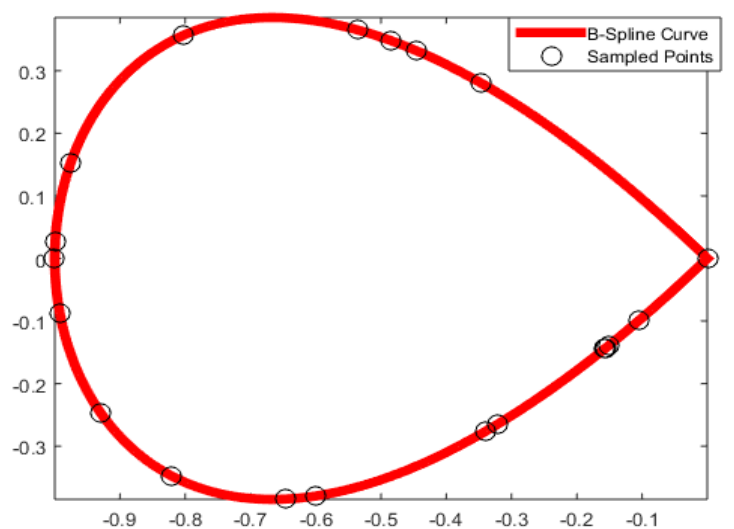

Fig. 9. B-Spline curve for Example-4 E. Example-5

The parametric curve equation $x(t)=u^{3}+u+2$

$y(t)=2 u^{3}-3 u^{2}-12 u+5$

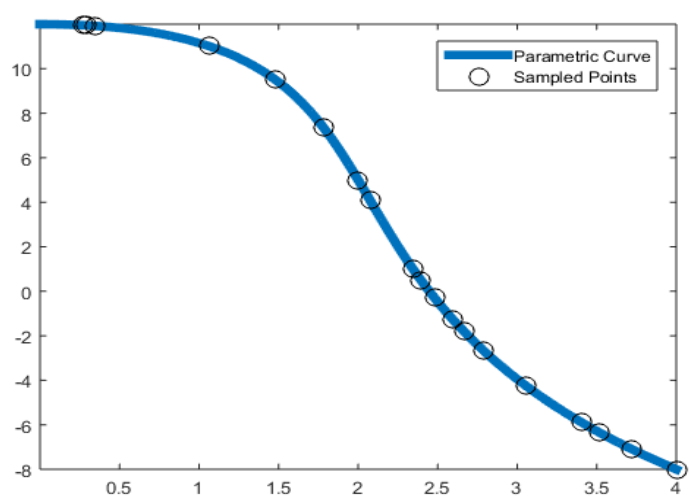

Fig. 10. Parametric curve for Example-5

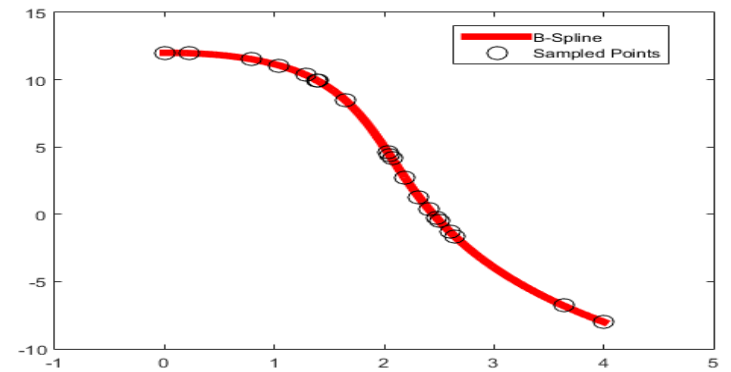

Fig. 11. B-Spline curve for Example-5

Published By:

Blue Eyes Intelligence Engineering \& Sciences Publication

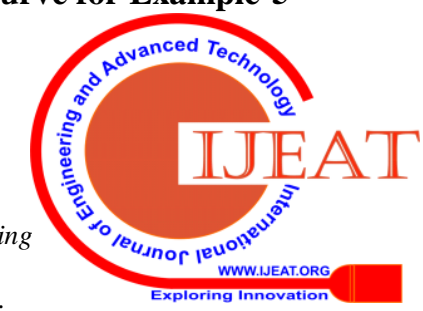


Example 3 consists of an implicit cuspoidal cubic. It was convereted into a parametric curve, and then to approximating B-Spline with the same parameter set used for other examples. From fig. 6 and fig. 7, it is evident that the algorithm captures the sampling points well and the same is supported by the results in table I. Curve with double point, example 4 shown in fig. 8, is approximated with a B-Spline with five control points, fig. 9. With only, 20 sampled points, acceptable accuracy levels were achieved. Capability of the algorithm to get accurate conversions was also demonstrated using example 5 and the results were as shon in fig. 10 and fig. 11.

\section{CONCLUSIONS AND FUTURE WORK}

The proposed algorithm for point sampling to convert planar parametric curve to B-Spline curve employs a hybrid fireworks algorithm for the identification of sampling points. It utilizes curvature information to identify the feature points. Then the fireworks algorithm is employed to identify the auxiliary points. As the algorithm uses randomly initialized parameter values and determine the auxiliary points in iterative manner to minimize conversion error, no user intervention is required for irregularly shaped curves. This makes the algorithm more general and results obtained for cuspoidal cubic indicates the same. Conversion errors obtained shows the potential of algorithm for the point sampling applications. As a part of the future work, the proposed algorithm will be applied for the spatial curves.

\section{ACKNOWLEDGMENT}

Authors are grateful to the Department of Mechanical Engineering of Godavari Institute of Engineering and technology for all the facilities given to carry out this work.

\section{REFERENCES}

1. L. Lu and S. Zhao, "High-quality point sampling for B-spline fitting of parametric curves with feature recognition," J. Comput. Appl. Math. vol. 345, pp. 286-294, 2019, doi: 10.1016/j.cam.2018.04.008.

2. C. Deng and X. Yang, "A local fitting algorithm for converting planar curves to B-splines," Comput. Aided Geom. Des., vol. 25, no. 9, pp. 837-849, 2008, doi: 10.1016/j.cagd.2007.11.001.

3. Y. Tan, Y. Tan, and Y. Zhu, "Fireworks Algorithm for Optimization Fireworks Algorithm for Optimization," no. December, pp. 355-364, 2015, doi: 10.1007/978-3-642-13495-1.

4. H. Park, "An error-bounded approximate method for representing planar curves in B-splines," Comput. Aided Geom. Des., vol. 21, no. 5, pp. 479-497, 2004, doi: 10.1016/j.cagd.2004.03.003.

5. L. Pagani and P. J. Scott, "Curvature based sampling of curves and 10.1016/j.cagd.2017.11.004.

6. W. Li, S. Xu, G. Zhao, and L. P. Goh, "Adaptive knot placement in B-spline curve approximation," CAD Comput. Aided Des., vol. 37, no. 8, pp. 791-797, 2005, doi: 10.1016/j.cad.2004.09.008.

7. L. Lu, "Sample-based polynomial approximation of rational Bzier curves,” J. Comput. Appl. Math., vol. 235, no. 6, pp. 1557-1563, 2011, doi: 10.1016/j.cam.2010.08.008.

8. L. Lu, "Weighted progressive iteration approximation and convergence analysis," Comput. Aided Geom. Des., vol. 27, no. 2, pp. 129-137, 2010, doi: 10.1016/j.cagd.2009.11.001.

9. D. J. Walton and D. S. Meek, "Curvature extrema of planar parametric polynomial cubic curves,” J. Comput. Appl. Math., vol. 134, no. 1-2, pp. 69-83, 2001, doi: 10.1016/S0377-0427(00)00529-X.

10. H. Yang, W. Wang, and J. Sun, "Control point adjustment for B-spline curve approximation," CAD Comput. Aided Des., vol. 36, no. 7, pp. 639-652, 2004, doi: 10.1016/S0010-4485(03)00140-4.

11. A. Razdan, "Knot Placement for B-Spline Curve Approximation," tech Rep. Arizona State Univ., 1999. surfaces," Comput. Aided Geom. Des., vol. 59, pp. 32-48, 2018, doi

12. C. Deng and H. Lin, "Progressive and iterative approximation for leas squares B-spline curve and surface fitting," CAD Comput. Aided Des., vol. 47, pp. 32-44, 2014, doi: 10.1016/j.cad.2013.08.012.

13. P. Laube, M. O. Franz, and G. Umlauf, "Learnt knot placement in B-spline curve approximation using support vector machines,' Comput. Aided Geom. Des., vol. 62, pp. 104-116, 2018, doi: 10.1016/j.cagd.2018.03.019

14. M. Kosters, "Cu rvatu re-dependent parameterization of curves and s u r f a c e s," vol. 23, no. 8, pp. 569-578, 1991.

15. H. Park, K. Kim, and S. C. Lee, "Method for approximate NURBS curve compatibility based on multiple curve refitting," CAD Comput. Aided Des., vol. 32, no. 4, pp. 237-252, 2000, doi 10.1016/S0010-4485(99)00088-3.

\section{AUTHORS PROFILE}

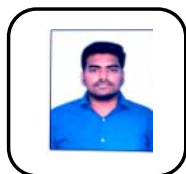

K Sreenivasa reddy is currently working as an Assistan professor in the Department of Mechanical Engineering of Godavari Institute of Engineering \& Technology, Rajahmundry. He obtained B.Tech degree in mechanical Engineering and M.Tech degree in manufacturing Engineering. His master's project is related to application of swarm intelligence algorithm for the fitting of Bezier and B-Spline curve to unorganized data sets. Before joining Godavari Institute of Engineering \& Technology, he started worked as Assistant professor in the Department of mechanical engineering in Dhanekula Institute of Engineering and Technology, Vijayawada. He teaches undergraduate courses of basic manufacturing sciences and basic introductory courses for freshers. His research interests include computer aided design and manufacturing advanced welding processes and metal additive manufacturing.

D Sai is an under graduate student of mechanical engineering at Godavari Institute of Engineering \& Technology, Rajahmundry. He obtained Diploma

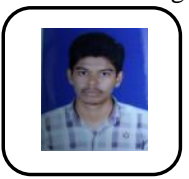
degree in mechanical engineering. His B.Tech min project is design and fabrication project on pesticides spraying machine carrier. His achieved AutoCAD certification from Autodesk and SOLIDWORKS certification from SEIMENS. He Did certification course on 3D part modelling in 3D EXPERIENCE and achieved CATIA certification from Dassault systems. His research interests include computer aided design and manufacturing, Design and ANSYS of different types mechanical parts and composite materials, advanced welding processes, swarm intelligence algorithm for curve conversion and fabrication of agriculture uses machines.

B Vijay Sai is an under graduate student of mechanical engineering at

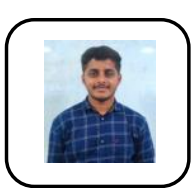
Godavari Institute of Engineering \& Technology, Rajahmundry. His B.Tech mini project is design and fabrication project on agriculture pesticides spraying machine carrier. His achieved ARC 1.02 .0 and 3.0 certification from ARC and did project based on this advanced car parking system programmable logic controller. And achieved AutoCAD certification from Autodesk and SOLIDWORKS certification in design from SEIMENS. His research interests include computer aided design and manufacturing, advanced robotic control systems, advanced welding processes, swarm intelligence algorithm for curve conversion and design and fabrication of agriculture uses machines for different applications.

E Kamal Raju is an under graduate student of mechanical engineering at

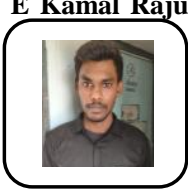
Godavari Institute of Engineering \& Technology, Rajahmundry. His B.Tech mini project is design and fabrication project on agriculture pesticides spraying machine carrier. His achieved AutoCAD certification from Autodesk. He Did certification course on Refrigeration and air conditioning based on refrigerants. His research interests include computer aided design and manufacturing Design and ANSYS of different types mechanical parts and composite materials, advanced welding processes, swarm intelligence algorithm for curve conversion and fabrication of agriculture uses machines, curve conversion by using different methods and algorithms based on the type of curve.

Published By: 
Hybrid Fireworks Algorithm for the Conversion of Planar Curve to B-Spline Curve

D Praveen Kumar is an under graduate student of mechanical engineering at Godavari Institute of Engineering \& Technology, Rajahmundry. His B.Tech mini project is design and fabrication project on pesticides spraying machine carrier. His achieved AutoCAD certification from Autodesk and SOLIDWORKS certification from SEIMENS. His research interests include computer aided design and manufacturing, Design and ANSYS of different types mechanical parts and interested in composite materials, advanced welding processes, swarm intelligence algorithm for curve conversion and fabrication of agriculture uses machines for different applications, curve conversion by using different methods and algorithms based on the type of curve and metal additive manufacturing.

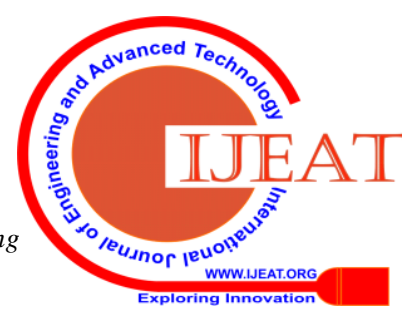

